Citation: D. Cino (2020) Dilemmi digitali e governance dell'identità digitale dei minori: l'interazione fra pari come opportunità informale di media education. Media Education 11(2): 149-161. doi: $10.36253 / \mathrm{me}-9027$

Received: June, 2020

Accepted: October, 2020

Published: December, 2020

Copyright: (c) 2020 D. Cino. This is an open access, peer-reviewed article published by Firenze University Press (http://www.fupress.com/me) and distributed under the terms of the Creative Commons Attribution License, which permits unrestricted use, distribution, and reproduction in any medium, provided the original author and source are credited.

Data Availability Statement: All relevant data are within the paper and its Supporting Information files.

Competing Interests: The Author(s) declare(s) no conflict of interest.

\section{Dilemmi digitali e governance dell'identità digitale dei minori: l'interazione fra pari come opportunità informale di media education}

\author{
Digital Dilemmas and governance of children's digital identities: \\ online communication as an (informal) opportunity for media \\ education
}

\section{DAvide Cino}

Università di Milano-Bicocca, Dipartimento di Scienze Umane per la Formazione/ Università Cattolica del Sacro Cuore, Milano, Dipartimento di Scienze della Comunicazione e dello Spettacolo

E-mail: davide.cino@unicatt.it

\begin{abstract}
The contribution investigates parents' digital dilemmas associated with sharenting as an informal learning opportunity to rethink and orient their online sharing behavior. The paper reports on some results of an exploratory study based on the analysis of online conversations on a parenting forum focused on these dilemmas. Findings suggest that the interactional context where such exchange takes place can function as an informal learning environment, fostering informal peer-to-peer media education practices for parents in the digital age.
\end{abstract}

Keywords: sharenting, digital dilemmas, informal learning, media education, social media.
Riassunto. Il presente contributo indaga i dilemmi digitali vissuti dai genitori rispetto alla pratica dello sharenting come occasione di apprendimento informale per ripensare ed orientare il proprio comportamento di condivisione online. Il paper riporta alcuni risultati di una ricerca esplorativa basata sull'analisi di conversazioni su un forum per genitori incentrate su questi dilemmi. I risultati suggeriscono che il contesto interattivo del forum in cui tali interazioni si dispiegano possa funzionare da ambiente informale di apprendimento, favorendo pratiche di educazione fra pari ai social media cui i genitori possono attingere nellera digitale.

Parole chiave: sharenting, dilemmi digitali, apprendimento informale, media education, social media. 


\section{INTRODUZIONE}

Gli ultimi anni hanno visto un incremento notevole nell'adozione dei media digitali all'interno del nucleo familiare, comportandone l'incorporazione in differenti prassi quotidiane. In questo contesto, gli studiosi hanno teorizzato il fenomeno del "digital parenting" (Mascheroni et al., 2018), designando con questa espressione l'eterogenea gamma di possibili adozioni dei nuovi media come estensione delle proprie pratiche genitoriali, quali l'utilizzo di forum, siti e applicazioni rivolte a genitori per "educarsi" alla genitorialità, strumenti per monitorare la prole (e.g. filtri per internet o app di geolocalizzazione), nonché la condivisione di foto e video di bambini sui social media, definita "sharenting" (Blum-Ross \& Livingstone, 2016).

Quest'ultima pratica, nel dettaglio, rappresenta il focus principale di questo contributo.

Tanto l'opinione pubblica quanto la letteratura scientifica hanno espresso opinioni spesso polarizzate in merito all'argomento, enfatizzando rischi e preoccupazioni connesse alla creazione di un'identità digitale dei minori, nonché alla possibilità per i genitori di pubblicare contenuti potenzialmente imbarazzanti per la prole e, in generale, compromettere la privacy del nucleo familiare (Siibak \& Traks, 2019).

Laddove le succitate preoccupazioni possano effettivamente costituirsi, almeno sul piano teorico, come "rischi" associati a questa forma di esposizione online, i dati empirici sull'argomento suggeriscono che la probabilità che tali rischi si trasformino effettivamente in conseguenze dannose per bambini e genitori è, ad oggi, piuttosto bassa e prevalentemente speculativa (Aroldi \& Mascheroni, 2019; Smahel et al., 2020).

Lo sharenting, tuttavia, è considerato un'espressione comunicativa normalizzata rientrante nella quotidianità di molte famiglie (Leaver, 2015). Benché la sua normalizzazione sia stata spesso letta presumendo un'aprioristica assenza di consapevolezza e riflessività da parte dei genitori (Barassi, 2019), diverse ricerche suggeriscono che nel valutare rischi e benefici associati al proprio comportamento di condivisione molti di loro vivano dei veri e propri "dilemmi digitali" (Blum-Ross \& Livingstone, 2017; Chalklen \& Anderson, 2017). La letteratura pedagogica supporta la valenza (tras)formativa del dilemma (Formenti \& West, 2018), intesa come opportunità di attivazione di pratiche critiche e riflessive dalle quali possono far seguito esperienze di apprendimento informali.

Su questa linea, il presente contributo indaga come tali dilemmi possano divenire occasioni di riflessione partecipata e apprendimento critico riportando alcuni risultati di una ricerca internazionale sull'utilizzo di un forum per genitori come arena di apprendimento informale da parte di utenti che hanno spontaneamente cercato il supporto dei pari online per affrontare dubbi e problematiche connesse allo sharenting. Nel farlo, considerò se e in che misura tale spazio comunicativo possa costituirsi come arena epistemica e morale in cui i genitori contemporanei possono educarsi reciprocamente all'utilizzo dei media in famiglia e apprendere ad esercitare la propria genitorialità nell'era digitale.

La peculiarità del lavoro poggia su due elementi principali. In primis, sull'utilizzo di dati naturali, ovvero conversazioni ordinare online in cui dei genitori, senza previa sollecitazione da parte di un ricercatore, hanno aperto una discussione tra pari per confrontarsi in merito a un dilemma vissuto. In secundis, sull'esplorazione del ruolo giocato dal forum per genitori come arena di discussione e apprendimento in merito a dilemmi morali e problematiche digitali, espandendo la letteratura sulla ricerca di supporto genitoriale online che, ad oggi, si è focalizzata su aree più tradizionali (quali dubbi e dilemmi inerenti al parto, l'educazione dei figli, scelte di natura pediatrica, etc. - Dworkin et al., 2013), tralasciando la ricerca di supporto rispetto all'incorporazione dei media digitali nel contesto familiare.

\section{LA GOVERNANCE DELLO SHARENTING: CONTROVERSIE E DILEMMI DIGITALI}

La governance della relazione tra bambini e media ha profonde radici storico-culturali che poggiano sulla figura moralmente connotata del "buon genitore" a cui è delegato tale compito (Caronia, 2010). La cultura occidentale pone particolare enfasi sul tema del rischio, rimarcando il ruolo preventivo e protettivo del genitore (Thomas et al., 2016), ed espandendo il range di panici e dilemmi morali a tutta una vasta gamma di aree che $\mathrm{i}$ genitori sono chiamati a controllare, tra i quali rientrano i media digitali. La letteratura suggerisce che madri e padri avvertano il peso di tale imperativo morale anche rispetto alla governance dello sharenting.

La peculiarità di tale pratica sta nel fatto che il bambino di cui si condivide potrebbe non giocare un ruolo attivo nel processo, pertanto non trarre alcun beneficio diretto da questa presenza online che diviene, di conseguenza, passiva.

Ciò è particolarmente vero nel caso di bambini ancora troppo giovani per dare il loro consenso alla condivisione online. In questo caso, la responsabilità di gestire e salvaguardare la loro presenza online ricade sugli adulti che condividono e, nella fattispecie, sui genitori, definiti i "gatekeepers" delle tracce digitali della 
prole (Steinberg, 2016). Tali specificità hanno comportato lo sviluppo di una serie di controversie associate alla pratica dello sharenting, etichettando spesso i genitori come irresponsabili e noncuranti delle potenziali problematicità del loro comportamento di condivisione (Barassi, 2019). La letteratura sull'argomento, tuttavia, mostra un aspetto più sottile del fenomeno, suggerendo che molti genitori, invece, cercano di governare la presenza dei figli sui social media stabilendo cosa non solo essi stessi, ma anche soggetti esterni alla famiglia nucleare, come i membri della famiglia allargata, possano o meno pubblicare (Ammari et al., 2015). Secondo uno studio di Chalklen e Anderson (2017), poi, molte madri vivono un "privacy/openness paradox", in cui se da un lato si apprezzano le opportunità personali derivanti dal proprio comportamento di condivisione, dall'altro se ne riconoscono i potenziali rischi o, quantomeno, l'assenza di benefici diretti per la prole. Allo stesso modo, Holloway e Green (2017) sottolineano come nel condividere foto familiari sui social, i genitori si confrontino con una serie di dilemmi riguardanti la permanenza e la sicurezza di queste rappresentazioni. La permanenza sul web di materiale ritraente la prole, inoltre, spinge molti ad interrogarsi in merito all'appropriatezza del contenuto condiviso e a prendere decisioni situazionali di conseguenza (Ammari et al., 2015).

Benché le ricerche documentino una dimensione dilemmatica che accompagna la pratica dello sharenting, la maggior parte degli studi che ad oggi ha trattato i dilemmi vissuti dai genitori non ha approfondito particolarmente come questi siano affrontati, né se e in che modo possano divenire occasione di apprendimento. L'assenza di modelli culturali di riferimento sull'argomento, nonché di supporto da parte delle generazioni precedenti a causa della novità del fenomeno (Leaver, 2015), implica che nell'interfacciarsi con una serie di dilemmi associati allo sharenting i genitori contemporanei possano sentirsi disorientati e bisognosi di supporto per meglio valutare i corsi d'azione da adottare. $\mathrm{Nel}$ prossimo paragrafo evidenzierò come il web possa ovviare a questa carenza di supporto, costituendosi come potenziale ambiente di apprendimento.

\section{IL WEB COME CONTESTO INFORMALE DI APPRENDIMENTO PER GENITORI}

Appurato che i media digitali e, nel dettaglio, la pratica dello sharenting costituiscano fonti di dilemmi genitoriali, illustrerò di seguito in che misura il web possa funzionare come contesto informale di apprendimento per affrontare questi dilemmi.
I genitori contemporanei, infatti, hanno incorporato i media digitali e, nel dettaglio, la rete nelle loro prassi quotidiane quali strumenti per cercare/offrire supporto e conforto e apprendere ad "esercitare" la propria genitorialità (Demozzi et al., 2020a). La nozione secondo cui la genitorialità possa essere appresa non è nuova nel dibattito pedagogico. A tal proposito, scrivono Formenti e West (2010),

quando si parla di apprendimenti in famiglia si parla soprattutto di processi informali, quotidiani, invisibili. I genitori, i figli, i prossimi, imparano continuamente gli uni dagli altri, dalle interazioni e dai feedback reciproci [...] più che da insegnamenti impartiti coscientemente (p. 35).

Il concetto stesso di genitorialità, nella sua nozione neoliberale, implica una postura del genitore come soggetto in costante apprendimento, in particolare alla luce delle possibili ansie e preoccupazioni derivanti dall'esercizio del suo ruolo di "buon genitore" (Lee et al., 2014). L'apprendimento della genitorialità, dunque, va inteso come un processo continuo che va oltre le circostanze educative formali, incorporando anche le prassi quotidiane che fanno capo a un più vasto processo di educazione sociale informale (Tramma, 2019). Esiste, infatti, un complesso processo di apprendimento permanente di natura informale, trasversale ai contesti di cui si fa esperienza e indipendente da un dato orientamento assiologico, tramite cui acquisiamo -anche inconsapevolmente e non intenzionalmente- una serie di nuove conoscenze, abilità, e valori a partire dall'esperienza quotidiana (Jarvis, 2012). Una tale concezione di apprendimento ci chiede tuttavia di interrogarci in merito al concetto stesso di "educativo". Secondo Tramma (2019), ad esempio, l'oggetto epistemico "educazione" è passibile di chiavi interpretative plurime, che possono tanto "dilatare" quanto "restringere" il campo semantico e di indagine che gli è proprio. Possiamo, cioè, reputare potenzialmente educativo - indipendentemente dall'assetto valoriale di riferimento- tutto ciò che ci circonda $\mathrm{o}$, al contrario, relegare l'educazione ai soli circuiti formali destinati ad erogarla.

In questo paper faccio riferimento al framework dell'apprendimento informale quale oggetto epistemico pedagogico che richiede una dilatazione della nostra comprensione di ciò che è considerato educativo. Uno sguardo che includa, cioè, tutte quelle esperienze di apprendimento che riguardano trasversalmente tutti i contesti e le esperienze di cui un soggetto fa esperienza che contribuiscono alla sua formazione continua, indipendentemente dalla loro intenzionalità formativa. Il presente contributo inquadra il web come un contesto di apprendimento informale. Questa scelta si svin- 
cola da un paradigma pedagogico che reputa educative solo le pratiche legate a contesti formali e orientate a un valore socialmente desiderabile, la cui trasmissione diviene finalità prima dell'azione educativa. Se invece, come sostiene Tramma (2019), educativo è tutto ciò che contribuisce $a$ (più che "comporta") un apprendimento di norme, valori, schemi interpretativi e sistemi di rappresentazione, la stessa esperienza educativa diviene qualcosa che rifugge il mero contenimento in contesti formalmente organizzati (ad es. la scuola) o socialmente intesi come educativi per antonomasia (ad es. la famiglia), incorporando l'esperienza di vita dei soggetti nel suo dispiegarsi in contesti molteplici e lungo tutto l'arco della vita, in linea con la prospettiva del lifelong/lifewide learning (Formenti \& West, 2010).

L'utilizzo di internet per affrontare le sfide della genitorialità rientra tra queste esperienze di apprendimento informale, grazie all'adozione di differenti piattaforme per genitori che offrono opportunità di apprendimento plurime e dalle modalità diversificate. Esempi di tali piattaforme sono siti internet istituzionali per genitori, forum basati su comunicazioni asincrone, blog, gruppi Facebook, gruppi WhatsApp, video su YouTube e pagine Instagram. Tra gli argomenti maggiormente rappresentati e dibattuti in questi spazi figurano la gravidanza, l'allattamento al seno, pratiche educative e alimentari da adottare con la prole, nonché la vita scolastica dei figli (Cino et al., 2020; Das, 2017; Pedersen \& Lupton, 2018). È tuttavia importante sottolineare che le ricerche suggeriscono una decisa connotazione di genere rispetto alla genitorialità online, con una forte preponderanza femminile nell'utilizzo di queste risorse e caratteristiche demografiche tipiche delle cosiddette famiglie "W.E.I.R.D." (Western, Educated, Industrialized, Rich, and Democratic - Dworkin et al., 2013; Henrich et al., 2010).

Ognuna delle piattaforme di cui sopra offre possibilità di azione e apprendimento differenziate. In questo paper mi concentrerò sui forum per genitori, valutando se e in che misura possano funzionare come comunità di pratica online (Wenger, 2011) dove i genitori possono attivare pratiche riflessive ed educative partendo dai loro dilemmi digitali.

\subsection{La specificità dei forum per genitori come arene epis- temiche}

I forum per genitori rappresentano un contesto interattivo particolarmente interessante da un punto di vista pedagogico. In primis, perché nonostante il proliferare di altre tipologie di piattaforme, tra cui le succitate, continuano a rappresentare una risorsa attivamente utilizzata da molti genitori (Lupton et al., 2016). Lo spa- zio interattivo che offrono, inoltre, permette a tutti gli utenti di aprire un thread che può poi essere indirizzato a un pubblico di pari i quali, generalmente, fanno parte di quella comunità online perché condividono scopi, problemi o condizioni comuni. Lo scambio comunicativo che ne risulta rappresenta una buona fonte di dati naturali che mettono in luce prospettive, percezioni personali e preoccupazioni delle parti interagenti (Holtz et al., 2012), consentendo ai genitori di esplicitare, discutere e apprendere dalle rispettive etno-teorie genitoriali (Harkness \& Super, 1992).

I forum per genitori, infatti, fanno parte del più vasto dominio delle community online, inquadrate da Petti (2011) come spazi di apprendimento informale che uniscono persone accomunate da un dato interesse e il cui funzionamento si basa sull'interazione sociale fra i membri che attivano processi ermeneutici e riflessivi tra le parti interagenti. Lautrice definisce le community online come contesti di apprendimento sociali, collaborativi e informali:

sociali perché sono costituti dalle relazioni che le persone instaurano tra loro, collaborativi perché la conoscenza è un fenomeno di costruzione e interazione sociale e informali perché si possono sviluppare apprendimenti inattesi derivanti dalla loro frequentazione (p. 39).

L'importanza delle pratiche riflessive quali antecedenti della costruzione identitaria genitoriale è un tema cruciale da un punto di vista pedagogico. Ora, è importante sottolineare che il più delle volte, nell'affrontare $i$ dilemmi e le sfide della vita familiare, i genitori possono fare riferimento esplicito o implicito, diretto o indiretto, a un ventaglio di copioni familiari (o script - Byng-Hall, 1998) in termini di aspettative condivise su cosa si debba o possa fare in determinate situazioni (Formenti, 2012). Tali copioni fanno parte di un insieme di saperi primari (Fabbri, 2008) che contribuiscono in larga parte alla costruzione del proprio sé genitoriale, nonché all'utilizzo di certi framework di riferimento per leggere una determinata situazione e valutare i corsi d'azione da intraprendere, e affondano le loro radici in quel corpus di esperienze che i genitori stessi hanno vissuto in precedenza in quanto figli nella famiglia di origine, osservando e partecipando ad attività di vita quotidiana. Esistono tuttavia circostanze in cui questi copioni si rivelano insufficienti, se non inadeguati. È il caso delle sfide poste da una nuova serie di problematiche che non trovano nell'esperienza pregressa un'adeguata cassa di risonanza. In un certo senso, i dilemmi digitali - soprattutto quelli associati a nuove pratiche mediatiche, come lo sharenting - fanno parte di queste esperienze prive di repertori passati e per le quali $\mathrm{i}$ genitori contemporanei possono trovarsi disorientati. 
Una delle più tradizionali forme di sostegno e apprendimento della genitorialità è proprio il confronto fra pari, che avviene spesso nell'ambito di conversazioni interpersonali informali, quali le chiacchiere fra genitori all'uscita dei bambini da scuola, durante una telefonata con amici e parenti ma anche, oggi, avvalendosi di conversazioni tecnologicamente mediate. $\dot{E}$ in questo senso che si può affermare che «i saperi genitoriali sono costruiti attraverso l'interazione quotidiana tra noi, gli altri, gli eventi» (Fabbri, 2008, p. 47), ed è nell'ambito di queste interazioni che si possono apprendere modalità di pensiero e azione che riecheggiano orizzonti di senso comune dati per scontati o a questi alternativi. In una logica non certa in termini di esiti, ma quantomeno potenziale, l'interazione sociale fra genitori può accompagnare una trasformazione del pensiero da pre-riflessivo, ovvero, che rispecchia in maniera acritica un insieme di assunti acquisiti e dati per scontati, a riflessivo, che guarda cioè più criticamente e analiticamente all'esperienza vissuta (Fabbri, 2008). Lo scambio tra prospettive differenti che può aver luogo nell'interazione fra pari, infatti, può favorire tali pratiche riflessive, promuovendo la creazione di nuovi margini di comprensibilità di un dato fenomeno con cui si è alle prese.

I forum per genitori, a tal riguardo, sono stati studiati in merito a una vasta gamma di dilemmi inerenti differenti aree della genitorialità, quali il parto, l'educazione dei figli, l'allattamento al seno, etc. (Dworkin et al., 2013). Poter raggiungere un pubblico di pari che vada oltre le proprie cerchie offline, nondimeno, dà ai genitori la possibilità di discutere argomenti per i quali fanno fatica a trovare supporto fra i tradizionali sistemi prossimali (Hine, 2014), potenziando così le opportunità di confronto e apprendimento.

Tale mancanza di tradizionali forme di supporto è di particolare interesse nel caso dello sharenting e dei media digitali in generale. Green e colleghi (2019) sottolineano, a tal proposito, le sfide senza precedenti che $\mathrm{i}$ genitori vivono nell'era digitale, affermando che il rapporto con i nuovi media è tra i compiti più complessi della genitorialità nella società contemporanea, vista la velocità con cui evolvono. Riprendendo Wenger (2011), gli autori evidenziano l'importanza delle comunità di pratica informali per genitori grazie alla possibilità di fornire uno scambio di esperienze dalle quali apprendere come affrontare le varie sfide poste dalla socializzazione digitale con cui le famiglie odierne si confrontano. Secondo Fabbri (2008), le comunità di pratica informali sono particolarmente importanti nel processo di costruzione identitaria genitoriale, in quanto consentono

la scoperta, da parte dei singoli, che altre persone affrontano problemi simili, condividono una passione per le stesse tematiche, possiedono esperienze, dati, strumenti, approcci, opinioni e idee rilevanti da mettere a disposizione e che c'è dunque occasione di apprendere molto l'uno dall'altro. [...] Formarsi significa potersi confrontare con altri genitori che hanno problemi simili, con genitori che hanno superato quei problemi. Si tratta in fondo di una formazione informale che già è largamente praticata e forse chiede di essere aiutata a coltivarsi. (pp. 54-55).

Wenger (2011) definisce le comunità di pratica come un gruppo di persone accomunate da un interesse (in senso esteso) che riguarda la loro vita quotidiana e rispetto al quale interagiscono e apprendono nuovi modi di fare, pensare ed essere. Questa definizione allargata, secondo l'autore, non implica necessariamente un'intenzionalità educativa, in quanto l'apprendimento può rappresentare tanto il fine per cui la comunità si crea, quanto una risultante informale e non predetta degli scambi interazionali fra i membri. Tre sono le caratteristiche che contraddistinguono una comunità di pratica: un dominio comune (inteso come campo tematico), una comunità e una pratica. Il dominio di interesse comune è ciò che distingue la comunità di pratica da un qualsiasi aggregato di individui. Far parte di una comunità di pratica, in questo senso, significa condividere una o più caratteristiche che accomunano i membri. La comunità è l'elemento che favorisce le attività condivise tra i membri, quali interazioni, discussioni e scambio di informazioni, promuovendo opportunità di apprendimento informale. La pratica, infine, fa riferimento alla condivisione -tramite l'interazione sociale- di uno specifico repertorio di risorse tipico di quella comunità, quali esperienze, storie, e corsi d'azione per affrontare determinati problemi.

Le comunità di pratica, sottolinea ancora Wenger (2011), possono assumere forme, dimensioni e strutture differenti, aver luogo sia offline che online, ed essere sia formali che completamente informali.

In tal senso, da un punto di vista teorico, un forum per genitori online può potenzialmente costituirsi come una comunità di pratica. Nella fattispecie, il dominio di riferimento è la genitorialità, soprattutto se intesa nella sua accezione neoliberale che enfatizza l'importanza del ruolo sociale del genitore in termini quasi professionalizzanti. La dimensione comunitaria, invece, è favorita dall'attività interattiva che contribuisce a processi di apprendimento e co-costruzione di significato. La pratica, infine, si basa sulla condivisione di storie che, una volta messe in circolo, possono dar luogo a specifici circoli ermeneutici (Gadamer, 1975).

A tal proposito, Wenger (2011) enuclea tra le attività tipiche delle comunità di pratica la richiesta di informazioni e la ricerca/scambio di esperienze tra membri. 
Queste due prassi sono particolarmente rilevanti nei forum per genitori in quanto, come sottolinea Lindholm (2017), fanno parte di una prassi narrativa online in cui degli utenti producono delle storie con il fine di meglio comprendere un'esperienza imparando dai propri pari, i quali sono chiamati a rispondere per stabilire un terreno di discussione comune al/dal quale accostarsi o discostarsi. Secondo l'autrice, le storie personali sono dapprima mobilitate nel messaggio di apertura (definito "problem message"), in cui l'utente che apre la discussione (d'ora in avanti "original poster" - OP) espone un dilemma per il quale chiede supporto, configurandosi come "advice-seeker", per poi essere discusso nei commenti degli altri utenti (d'ora in avanti "commenting posters" - CPs), i quali possono a loro volta mobilitare le rispettive storie, esperienze ed opinioni, configurandosi come "advice-givers". La narrazione, dunque, favorisce la traduzione di un problema privato in linguaggio collettivo all'interno del forum, che diviene uno spazio informale «per potersi raccontare» (Formenti, 2010, p. 7).

In questo contesto, l'interazione funziona in prima battuta come attività di "problem posing", promuovendo uno scambio di opinioni ed esperienze che può potenzialmente educare le parti interagenti e favorire -ma non determinare- delle opportunità di apprendimento informale ed eventualmente di "problem solving", non soltanto per chi partecipa attivamente alla discussione, ma anche per chi si limita a leggerla sul momento o in futuro. La persistenza del materiale prodotto e pubblicato online, infatti, facilita la creazione di depositi di esperienze informali le cui potenzialità di apprendimento valicano i confini spazio-temporali dell'interazione offline, costruendo un corpus di conoscenze a disposizione di altri genitori che si trovano o potrebbero trovarsi a vivere una situazione dilemmatica analoga a quella discussa.

La richiesta di supporto in un forum per genitori, nonché lo scambio interattivo che da tale richiesta muove, possono infine essere compresi in riferimento all'autorità epistemica etero- ed auto-attribuita delle parti interagenti, in linea con la natura -empiricamente supportata- dei forum online come "arene epistemiche" (Landqvist, 2016). Il processo di costruzione della conoscenza, infatti, è eminentemente sociale e dipende a un tempo dalle conoscenze che apprendiamo dagli altri e dalle nostre esperienze, nonché da quelle che noi stessi produciamo e mettiamo in circolo interagendo con terze parti (Kruglanski et al., 2009). Kruglanski e colleghi (2009), a tal proposito, evidenziano come la conoscenza del singolo soggetto in merito a un certo dominio di esperienza possa essere deficitaria, spingendo pertanto a cercare supporto conoscitivo in terzi, definiti "knowledge providers". Il costrutto dell'autorità epistemica etero- attribuita è qui di particolare rilievo, in quanto fa riferimento a una fonte di conoscenza reputata credibile cui attingere quando si avverte un bisogno di apprendimento. La credibilità della fonte non dipende necessariamente dall'oggettività informativa di cui tale fonte può farsi portavoce, quanto da una combinazione di expertise percepita e fiducia da parte del soggetto che a quella fonte si è rivolto. Diversi sono, secondo gli studiosi, gli elementi che possono sancire l'autorità epistemica, quali un ruolo sociale (ad es. essere un genitore, uno psicologo, un insegnante), il livello di istruzione, l'istituzionalità di un enunciato, etc. (Kruglanski et al., 2009).

Strettamente interconnesso è il concetto di autorità epistemica auto-attribuita, indicante quanto il singolo soggetto si senta competente in merito a un determinato argomento. La richiesta di supporto conoscitivo è tanto più ritenuta necessaria quanto maggiore un individuo reputerà lo scarto tra la propria conoscenza su un argomento e quella altrui (Kruglanski et al., 2009).

$\mathrm{Nel}$ caso specifico del forum per genitori, l'autorità epistemica dei membri è data dalla stessa appartenenza alla categoria di genitori (presenti o futuri) quali insider dell'esperienza genitoriale e depositari di determinate conoscenze che, messe in circolo, possono contribuire al funzionamento della comunità di pratica favorita dagli scambi interattivi resi possibili dal forum.

A seguito di questa premessa teorica, il prossimo paragrafo indagherà se a che livello i forum genitoriali possano -nella loro declinazione di comunità di praticafavorire processi informali di educazione all'utilizzo dei media tra pari che supportino i genitori ad affrontare $i$ dilemmi digitali con cui si interfacciano.

\section{UN FORUM ONLINE COME OPPORTUNITÀ DI MEDIA EDUCATION? OBIETTIVI E RISULTATI (PARZIALI) DI UNA RICERCA}

Sulla base delle premesse di cui sopra, riporterò di seguito alcuni risultati parziali del progetto di ricerca internazionale "Sharenting and the datafication of family life: exploring parents' Social Media Dilemmas", che ha studiato i dilemmi digitali associati alla pratica dello sharenting discussi dai genitori sulla sezione pubblica del forum BabyCenter, reputato uno dei forum genitoriali maggiormente usati nel mondo occidentale prevalentemente da madri (Lupton et al., 2016). Il progetto ha indagato tanto le tipologie di dilemmi riportati dai posters, quanto le interpretazioni e soluzioni co-costruite dalle parti interagenti nel commentare e discutere questi dilemmi. Visto il focus di questo studio sulla sezione statunitense del forum, i dati qui riportati sono 
da intendersi in ottica contestuale e situazionale, ovvero non generalizzabili e passibili di specificità tipiche di quel determinato milieu socio-culturale.

Il campione dello studio allargato su cui poggia questo lavoro è stato raccolto utilizzando il motore di ricerca interno al sito tramite l'adozione di un'apposita stringa di parole chiave inerenti al topic di ricerca. È stato seguito un approccio di raccolta dati sequenziale di tipo top-down (Eriksson \& Salzmann-Erikson, 2013), passando in rassegna le prime 150 pagine di risultati, filtrando solo quelle discussioni incentrate sui dilemmi dello sharenting, in linea con ricerche precedenti che hanno adottato metodologie analoghe (Pedersen \& Lupton, 2018).

Nel presente contributo, mi concentrerò sull'analisi di una discussione (i.e. un "thread") come studio di caso con l'obiettivo di indagare:

1. se e in che misura la pratica conversazionale online possa costituirsi come opportunità di educazione informale all'uso dei media;

2. che tipo di messaggi impliciti ed espliciti vengono mobilitati dalle parti in causa rispetto all'essere genitori nell'era digitale.

Il focus su un singolo thread è supportato nella letteratura metodologica sullo studio dei forum online quando l'analisi mira a cogliere la specificità, più che la generalizzabilità del dato (Jowett, 2015). Pertanto, i risultati di seguito riportati sono da intendersi in ottica situazionale e contestuale. È inoltre importante sottolineare che, nonostante la natura anonima delle interazioni (in quanto gli utenti utilizzano degli usernames), indizi contestuali (quali gli stessi nicknames o l'uso di pronomi femminili) suggeriscono che le parti interagenti fossero donne, in linea con la forte preponderanza femminile tipica dei forum per genitori riportata in letteratura e il pubblico di madri a cui il forum in questione è prevalentemente rivolto (Dworkin et al., 2013; Lupton et al., 2016).

Di seguito ripoterò alcuni risultati di un'analisi tematica (Riessman, 2008) che -informata dalla succitata struttura interattiva delle discussioni online proposta da Lindholm (2017) - ha ricercato nel testo il tipo di dilemma riportato dall'original poster, le traiettorie ermeneutiche e discorsive proposte dai commenting posters, nonché indizi di trasformazioni e cambiamenti di prospettiva da parte dell'original poster a seguito dello scambio interattivo. La tematizzazione dei dati (Saldaña, 2009) ha avuto il fine di evidenziare i pattern tematici individuati nella discussione rappresentanti il prodotto $e$ dell'attività interazionale delle parti interagenti $e$ dell'esercizio ermeneutico del ricercatore che li ha letti e interpretati in una logica mista induttivo-deduttiva, tanto ancorata al dato, quanto informata da appositi fra- meworks teorici. L'analisi ha beneficiato dell'aiuto di due assistenti di ricerca che hanno volontariamente partecipato alla codifica della conversazione. Nel dettaglio, il primo step dell'analisi si è basato su una codifica iniziale da me condotta per familiarizzare con i dati e costruire una lista di codici che è stata poi accorpata in codici di secondo livello. Ciò ha portato alla stesura di un libro codice provvisorio, che è stato testato indipendentemente dalle assistenti di ricerca, le quali hanno fornito feedback, aggiustamenti e aggiunte. Il corpus di dati è stato infinite ricodificato alla luce dell'aggiornamento del libro codice e i risultati sono stati organizzati in modo tale da evidenziare la natura del dilemma, le traiettorie interpretative e pragmatiche costruite nello scambio interazionale e le possibili ridefinizioni e cambiamenti di prospettiva dell'original poster a seguito dell'interazione.

\subsection{Il caso delle "bathtime photos": costruire e apprendere modelli di "buona" genitorialità digitale}

Il thread qui analizzato è stato scritto da una madre che, dopo aver pubblicato una foto del figlio durante il bagno (in cui non erano visibili le parti intime), ha ricevuto delle critiche da parte dei suoi stessi genitori (i.e. i nonni del bambino), accusandola di aver tenuto un comportamento inadeguato. La madre, pertanto, si è rivolta alla comunità del forum raccontando l'accaduto, esprimendo opinioni e stati d'animo e chiedendo l'intervento dei pari sia tramite commenti che attraverso l'utilizzo di un sondaggio. Il sondaggio in questione, nella fattispecie, chiedeva se pubblicare "bathtime photos" fosse inopportuno o meno, fornendo le seguenti due opzioni di risposta:

1. Yes, you never know what can happen with that photo;

2. No, it's fine as long as privates are covered.

In totale, 219 utenti hanno risposto al quesito, di cui il 56\% ha votato per la prima opzione, e il rimanente $44 \%$ per la seconda. Il sondaggio viene qui mobilitato come strumento di consultazione veloce e diretto per ingaggiare altri genitori nella discussione e avere una metrica delle loro opinioni. La figura 1 sottostante riporta l'estratto di apertura dell'original poster $(\mathrm{OP})$ :

In linea con Lindholm (2017), il post di apertura dell'OP svolge la funzione di problem posing: la madre in questione, infatti, narra la sua esperienza fornendo una serie di elementi contestualizzanti atti a definire la situazione dilemmatica per la quale sta cercando aiuto. In primis, l'OP offre delle informazioni di background (l'aver pubblicato delle foto del figlio durante il bagno) e riporta il problema (la reazione dei nonni del bambino). Il giudizio negativo nei confronti della madre - il cui 


\section{Original Poster:}

So, I posted some pictures of my son during bathtime on Facebook today. You could hardly see his privates in the photo anyway but everything you could see I covered it with an emoji.

Well, I got a text from my dad asking me to take the photos down. My mom called me and told me the same thing.

They say it is because of predators and someone I'm friends with on Facebook can sell the photos on the dark web???

They both upset me very much. I realize that there are sick perverts out there and I would never put my son in harm, but I feel like they're being paranoid as hell. Not to mention I'm so upset with how they attacked me and told me what to do.

So, what do you guys think? How should I deal with this?

\section{BACKGROUND}

\section{PROBLEMA}

RISCHIO ONLINE: STRANGER-DANGER

\section{CONFERMA IDENTITÀ} "BUONA MADRE"

DILEMMA

Figura 1. Post di apertura - presentazione del dilemma.

ruolo morale di buona genitrice viene messo in discussione - è informato da un framework di riferimento tipico dei discorsi sui bambini online: lo stranger-danger (Jewkes \& Wykes, 2012). Questa figura, ricorrente in narrazioni allarmistiche rispetto alla presenza digitale dei minori, designa la possibilità che le foto del bambino finiscano in mani sbagliate, tradendo il ruolo socialmente atteso della madre di prevenire questo rischio; ruolo che la madre, al contrario, sente di rispettare ("I would never put my son in harm"). Il post si chiude con una domanda diretta indirizzata alle utenti del gruppo, la cui expertise come madri -e la relativa autorità epistemica che ne deriva- vengono chiamate in causa per meglio comprendere ("what do you guys think?) ed affrontare ("How should I deal with this?") la situazione. La richiesta di commenti scritti e l'uso del sondag- gio di cui sopra funzionano come detonatori di opinioni e conoscenze tra pari che trovano nel forum uno spazio informale per essere condivise ed eventualmente apprese come potenziali framework di riferimento e corsi d'azione (Das, 2017).

I commenti lasciati dalle utenti del forum (Commenting Posters - CP), che ricoprono qui il ruolo di $a d v i$ ce-givers, hanno risposto alla richiesta dell'OP seguendo due traiettorie differenti: da un lato una traiettoria problematizzante e dall'altro una traiettoria de-problematizzante (Cino \& Dalledonne Vandini, 2020). Le figure 2 e 3 ne riportano alcuni esempi accorpati in base alla loro natura ermeneutica (i.e. interpretativa della situazione riportata dall'OP).

La traiettoria problematizzante (Fig. 2) enfatizza i rischi dello sharenting e il ruolo protettivo che una

$C P_{\text {I: }}$ Just my opinion but I don't think bathtime photos should be posted on social media. There are plenty of cute photos in other environments where the child is covered with clothes that a tub photo doesn't need to be shared. In a photo album, like a physical album, okay, but I don't feel like it's necessary to post it online.

$C P_{2:}$ I agree with your parents. Do you post pictures of yourself in the tub/shower? You have a choice. Your baby relies on you to make good decisions. The way the internet and people are today, I want as few pics as possible online.

TRAIETTORIA

PROBLEMATIZZANTE

$C P_{3}$ : Just my opinion, but I wouldn't post it at all. There are sick people out there and my husband and I post very limited pictures of our son, never bathtime. Having said that, I wouldn't appreciate someone telling me what to do with my child or what to post so you can probably tell your parents that you thank them for their concern but that you and your partner will decide what goes on FB or not.

Figura 2. Commenti "problematizzanti" - interpretazioni e corsi d’azione. 
$C P_{4}$ : I know I'm in the minority here, but I don't think it's a big deal. To answer if I would post a bath pic of myself, the answer is yes. This [foto pubblicata] was a profile picture of myself 2 years ago. Everything is covered, but I'm still in a bathtub. Personally, I haven't posted a bath pic of my son, but that's only because I've only posted around five pics in total of him. I'm not a big sharer.

$C P_{5}$ : I think you should post what you want. There are sexual predators everywhere even in public. Are you all going to cover your children head to toe when you go to the beach or pool? If you want to post pics in the bath or be naked, go ahead.

$\mathrm{CP}_{6}$. Oh geez! To be honest, any picture of your child on social media is up for some sick person to be into. As long as no private parts are showing I don't see the big deal. Geez tell your parents to get their panties out of a wad

Figura 3. Commenti “de-problematizzanti” - interpretazioni e corsi d'azione.

"buona" madre è chiamata a ricoprire $\left(\mathrm{CP}_{2}\right.$ - "Your baby relies on you to make good decisions"). Tale prospettiva è in linea con il concetto di "problematizzazione" proposto da Lee et al. (2014), secondo cui la genitorialità neoliberale poggia le sue basi su un discorso sociale per cui salvaguardare il benessere dei figli rappresenti la conferma per un genitore di un corretto assolvimento a un suo precipuo dovere.

Il linguaggio usato dai CPs nella traiettoria problematizzante riflette tale aspettativa. Viene messa in campo una pedagogia del "dover essere" e del sottile biasimo, che porta a enfatizzare la problematicità del comportamento materno. L'utilizzo del condizionale $\left(\mathrm{CP}_{1}\right.$ "I don't think bathtime photos should be posted"; $\mathrm{CP}_{3}$ "I wouldn't post it at all") e delle domande dirette rivolte all'OP $\left(\mathrm{CP}_{2}\right.$ : "Do you post pictures of yourself in the tub/shower?") rimarcano ulteriormente tale pedagogia, suggerendo implicitamente non solo il quadro interpretativo (quello della "buona madre"), ma il corso d'azione adeguato da adottare (ovvero, non pubblicare le foto del figlio).

Viceversa, la traiettoria de-problematizzante (Fig. 3), minimizza il concetto di rischio rischio e lo sottrae al controllo genitoriale, inquadrandolo come ingovernabile $\left(\mathrm{CP}_{6}\right.$ "any picture of your child on social media is up for some sick person to be into"); inoltre mette in discussione il ruolo della "buona" madre socialmente atteso ( $\mathrm{CP}_{4}$ "I don't think it's a big deal"), rimarcando piuttosto l'agency dell'OP nel decidere se e cosa pubblicare del figlio ( $\mathrm{CP}_{5}$ "you should post what you want"). La conferma e messa in discussione del paradigma della buona genitorialità che ha qui luogo è ulteriormente supportata da studi precedenti condotti su forum per genitori simili (Pedersen, 2016), dimostrando come l'interazione sociale online consenta non soltanto di rinforzare, ma anche di de-costruire modelli pedagogici dati per scontati.

La polarizzazione delle due traiettorie ermeneutiche qui presentate, inoltre, ripropone la natura essenzialista dei discorsi sul rapporto tra bambini e media digitali (Mascheroni, 2018), contribuendo alla creazione di quelli che Das (2017) ha definito "mediated frameworks of reference" quali lenti attraverso cui guardare a un problema e apprendere possibili chiavi interpretative e corsi d'azione da seguire.

La figura 4, ritraente la risposta dell'OP, esemplifica la reazione della madre in questione allo scambio interattivo creatosi con i pari, offrendo supporto per la nozione che la comunicazione online può contribuire a dei processi di apprendimento informale, nella fattispecie rispetto all'utilizzo dei media in famiglia.

$O P$ : Thank you everyone for the input! I appreciate all the answers, even the condescending ones lol. I decided to take the photos down. Even though I don't find baby bathtime photos creepy or even somewhat close to an adult posting a photo of themselves in the bath, the more I thought about it you never know what can happen with a photo online these days and I would rather be safe than sorry. Now to just speak to my parents about not telling me what to do....

TRASFORMAZIONE DELLA PROSPETTIVA INIZIALE

Figura 4. Risposta dell’Original Poster. 
Comparando la posizione dell'OP tra il post di apertura e la risposta notiamo una trasformazione della prospettiva iniziale, evidente nel passaggio da "they're being paranoid as hell" -riferito alla reazione dei nonni del bambino- (Fig. 1) a "you never know what can happen with a photo online these days and I would rather be safe than sorry" (Fig. 4). Si osserva come la postura dell'OP rifletta adesso la posizione maggioritaria del sondaggio, rinforzata dai commenti improntati a una traiettoria problematizzante che sembrano aver contribuito alla decisione di rimuovere le foto pubblicate. L'adesione all'imperativo morale della "buona madre" può essere qui intesa in termini di supporto di una modalità di comportamento socialmente accettabile e "responsabile", proponendosi come modello culturale da apprendere e introiettare nelle proprie prassi di genitorialità digitale. Anche la traiettoria de-problematizzante, tuttavia, pare aver avuto un'incidenza, supportando la scelta dell'OP di parlare con i suoi genitori per reclamare la sua agentività nella governance della presenza digitale del figlio ("now just to speak to my parents about not telling me what to do").

Complessivamente, la conversazione qui analizzata suggerisce come lo spazio interattivo offerto dal forum abbia consentito l'attivazione di un circolo ermeneutico e riflessivo avente come oggetto epistemico la pratica dello sharenting, attorno al quale sono state espresse e convalidate posizioni differenti che, nel loro insieme, hanno co-costruito dei possibili quadri interpretativi più ampi rispetto alla prospettiva offerta inizialmente $\mathrm{e}$ hanno suggerito diversi possibili corsi d'azione da adottare a disposizione non soltanto dell'OP, ma di qualsivoglia genitore dovesse trovarsi in una situazione simile. In tal senso, l'analisi supporta che, a determinati livelli e in ottica non deterministica, lo scambio interattivo qui presentato possa funzionare come opportunità di media education informale per costruire e apprendere modelli di genitorialità digitale che possono rinforzare oppure mettere in discussione il modello pedagogico-culturale del "buon genitore".

\section{CONCLUSIONI}

Il contributo ha messo in luce come e in che misura un forum per genitori, qui inteso come comunità di pratica online, possa costituirsi come ambiente informale di apprendimento che favorisca processi di media education fra pari. Riportando, a scopo esemplificativo, i risultati dell'analisi di un thread in cui veniva discussa dalle parti interagenti la legittimità, per un genitore, di condividere contenuti potenzialmente imbarazzanti per la prole online, si è visto come lo spazio conversaziona- le offerto dal forum, nonché la struttura interattiva del thread, abbiano favorito uno scambio in cui erano evidenti tanto la presentazione di un dilemma rispetto al quale l'OP presentava un bisogno di supporto e formativo (i.e. apprendere come leggere la situazione e come comportarsi), quanto la pletora di interpretazioni e corsi d'azione proposti dai pari che hanno sostenuto una ridefinizione e trasformazione di prospettiva nell'OP. Questi elementi, insieme alla costruzione sociale di un deposito permanente di conoscenza online, supportano la tesi secondo cui i forum per genitori, quali spazi interattivi, possono effettivamente funzionare da comunità di pratica, offrendo opportunità di apprendimento informale che veicolano ideali e conoscenze rispetto all'essere genitore nell'era digitale. L'esperienza del dilemma come circostanza disorientante da cui possono emergere opportunità formative e trasformative è riportata nella letteratura pedagogica sull'apprendimento in età adulta (Mezirow, 1991). Poco si sa, tuttavia, in merito ai processi di apprendimento e costruzione identitaria che hanno a che fare con la genitorialità e, nel dettaglio, con l'esercizio della propria genitorialità digitale. Questo paper vede negli scambi interattivi fra questo gruppo di madri un'occasione di educazione informale all'utilizzo dei media in famiglia. Nel farlo, propone un framework di riferimento che riconosce l'assenza di intenzionalità formativa pre-costituita quale fattore caratterizzante tali conversazioni che si pongono come occasioni di co-educazione e co-costruzione di significato rispetto alla pratica mediatica dello sharenting. Laddove gli interventi di educazione ai media formalmente costituiti partono dal riconoscimento di un bisogno formativo, la definizione di certi obiettivi e la valutazione, in termini di outcome, dell'intervento stesso, l'esperienza qui riportata mostra un'esperienza educativa e di apprendimento complementare a quelle formali ma da queste differente. Il dilemma può essere letto come manifestazione di un bisogno da un punto di vista formativo (e.g. "come comportarmi rispetto alla pratica dello sharenting?"). La mobilitazione di risorse narrative e interattive, nonché la co-costruzione di framework di significato tra pari, invece, rappresenta una risposta "spontanea" (i.e. non etero-diretta) a tale bisogno. La conversazione informale online tra genitori, in questo caso rispetto al dominio della genitorialità digitale, diviene dunque un'opportunità di apprendimento informale nell'ambito di un contesto online -i.e. il forum in questione- che si costituisce come ambiente di apprendimento informale tra pari. Il desiderio di confrontarsi e apprendere come leggere e affrontare i dilemmi digitali vissuti, inoltre, è in linea con la nozione di "social media literacy" connessa al concetto di 
parental mediation, in riferimento alle conoscenze e competenze dei genitori messe in campo per governare la presenza digitale della prole, nonché al loro desiderio di acquisirle (Daneels \& Vanwynsberghe, 2017).

E tuttavia importante sottolineare che le opportunità formative qui discusse possono essere limitate a un gruppo elitario di genitori: il comunicare online sull'esperienza di condivisione online è, infatti, una prassi metariflessiva che richiede non soltanto un accesso costante alla rete, ma anche un grado di consapevolezza in merito a questioni digitali che non tutti i genitori possono condividere. Un ulteriore elemento che bisogna tenere in mente, inoltre, è la natura situazionale e contestuale di questi risultati. Aver raccolto dei dati su una piattaforma statunitense, infatti, ci chiede di contestualizzare le informazioni e interpretazioni qui riportate in riferimento al contesto nord-americano, che benché possibilmente risonante con altri contesti occidentali non è in sé per sé passibile di generalizzazioni. La letteratura, infatti, suggerisce che la cultura nord-americana promuove una nozione di "genitorialità intensiva" che pone particolare enfasi sulle responsabilità del "buon genitore" chiamato ad apprendere come esercitare il proprio ruolo sociale per garantire protezione alla prole (Shirani et al., 2012). I dati qui analizzati suggeriscono che tale responsabilità può oggi trovare estensione anche rispetto al ruolo del "buon" genitore digitale (Mascheroni et al., 2018). Le ipotesi interpretative qui avanzate, tuttavia, sono da intendersi valide tenendo presente l'indicalità tipica di tale processo ermeneutico, tanto rispetto alla soggettività interpretativa del ricercatore, quanto alle unicità socioculturali dei dati (Caronia, 1997).

Cionondimeno, è qui rilevante sottolineare come la possibilità per più parti interagenti di confrontarsi online su temi inerenti ai media in famiglia rappresenti un'opportunità per esercitare capacità meta-riflessive quali occasioni di media education fra pari, in cui i media digitali possono costituirsi non soltanto come causa di un dato dilemma, ma anche come possibile soluzione. Ciò è tanto più rilevante se si considera l'assenza di modelli culturali di riferimento in merito alla digitalizzazione continua della vita familiare. Un'attività ermeneutica come quella qui riportata mostra come tali modelli possano essere attivamente co-costruiti e appresi dai genitori contemporanei, tanto rinforzando quanto andando oltre la dicotomia giusto/sbagliato che spesso accompagna i discorsi sulle "buone" prassi di educazione ai media. I risultati di questo lavoro suggeriscono che tali prassi non riguardano solo il terreno dell'educazione formale e intenzionale, ma possono anche rientrare a pieno titolo nello studio dei nuovi media attraverso le lenti teoriche dell'educazione informale e degli adulti. Nel farlo, tutta- via, possono rivelarsi altresì informativi rispetto all'ideazione e progettazione di occasioni formative formali e non formali. L'esperienza del dilemma, infatti, rappresenta un importante monito rispetto a un bisogno formativo possibilmente avvertito dalle famiglie contemporanee in merito all'incorporazione di certe pratiche mediatiche quali lo sharenting- nel contesto domestico. In questo senso, appositi spazi (offline e online) possono essere ideati per favorire processi di riflessività in merito all'uso dei media in famiglia, così da ampliare il ventaglio di opportunità educative per i genitori contemporanei rispetto alle loro esperienze di genitorialità digitale.

\section{RINGRAZIAMENTI}

L'autore ringrazia le assistenti di ricerca Carlotta Bagnasco e Valentina Costa, studentesse all'Università di Milano-Bicocca, per il supporto offerto nella fase di codifica dei dati, nonché per i preziosi suggerimenti e scambi scaturiti da questo processo.

\section{BIBLIOGRAFIA}

Ammari, T., Kumar, P., Lampe, C., \& Schoenebeck, S. (2015). Managing Children's Online Identities. Proceedings of the 33rd Annual ACM Conference on Human Factors in Computing Systems - CHI '15, 1895-1904.

Aroldi, P., \& Mascheroni, G. (2019). Oltre il cyberbullismo. Il contributo di eu kids online alla ricerca sul benessere sociale dei giovani utenti della rete. Media Education, 10(2), 181-193. doi:10.14605/ MED1021903.

Barassi, V. (2019). Against Sharenting. Child Data Citizen. http://childdatacitizen.com/against-sharenting/

Blum-Ross, A., \& Livingstone, S. (2017). "Sharenting," parent blogging, and the boundaries of the digital self. Popular Communication, 15(2), 110-125. https:// doi.org/10.1080/15405702.2016.1223300

Byng-Hall, J. (1988). Le trame della famiglia. Attaccamento sicuro e cambiamento sistemico. Raffaello Cortina.

Caronia, L. (1997). Costruire la conoscenza. Interazione e interpretazione nella ricerca in campo educativo. La Nuova Italia.

Caronia, L. (2010). The family's governance of children's media consumption as a moral arena: theoretical framework, methodology and first results of a study. $R P D$ - Journal of Theories and Research in Education, 5(1), 1-20. https://doi.org/10.6092/issn.19702221/1741 
Chalklen, C., \& Anderson, H. (2017). Mothering on Facebook: Exploring the Privacy/Openness Paradox. Social Media + Society, 3(2) 1-10. https://doi. org/10.1177/2056305117707187

Cino D., \& Dalledonne Vandini, C. (2020). "Why does a teacher feel the need to post my kid?" Parents and Teachers Constructing Morally Acceptable Boundaries of Children's Social Media Presence. International Journal of Communication, 14, 1153-1172.

Cino D., Gigli A., \& Demozzi S. (2020). "That's the only place where you can get this information today!" An exploratory study on Parenting WhatsApp Groups with a sample of Italian parents. Reladei - Revista Latinoamericana de Educacion Infantil, in pubblicazione.

Daneels, R., \& Vanwynsberghe, H. (2017). Mediating social media use: Connecting parents' mediation strategies and social media literacy. Cyberpsychology: Journal of Psychosocial Research on Cyberspace, 11(3), article 5. https://doi.org/10.5817/CP2017-3-5

Das, R. (2017). Speaking About Birth: Visible and Silenced Narratives in Online Discussions of Childbirth. Social Media + Society, 3(4), 1-11. https://doi. org/10.1177\%2F2056305117735753

Demozzi, S., Gigli, A., \& Cino, D. (2020a). I media digitali come strumenti per "esercitare e performare" la genitorialità (parte 1): literature review e presentazione della ricerca. Rivista Italiana Di Educazione Familiare, 15(2), 79-92. https://doi.org/10.13128/rief7422

Demozzi, S., Gigli, A., \& Cino, D. (2020b). I media digitali come strumenti per "esercitare e performare" la genitorialità (parte 2): analisi e discussione dei risultati. Rivista Italiana Di Educazione Familiare, 15(2), 93-111. https://doi.org/10.13128/rief-7423

Dworkin, J., Connell, J., \& Doty, J. (2013). A literature review of parents' online behavior. Cyberpsychology, 7(2), 1-10. doi:10.5817/CP2013-2-2

Eriksson, H., \& Salzmann-Erikson, M. (2013). Supporting a caring fatherhood in cyberspace - an analysis of communication about caring within an online forum for fathers. Scanidavian Journal of Caring Science, 27, 63-69. Doi: 10.1111/j.1471-6712.2012.01001.x

Fabbri, L. (2008). Il genitore riflessivo. La costruzione narrativa del sapere e delle pratiche genitoriali. Rivista Italiana di Educazione Familiare, 45-55. https:// doi.org/10.13128/RIEF-3072

Formenti, L. (2010). La famiglia dalla solitudine all'agorà. Animazione sociale, 244, 34-41.

Formenti, L. (Ed.). (2012). Re-inventare la famiglia. Guida teorico-pratica per i professionisti delleducazione. Maggioli Editore.
Formenti, L., \& West, L. (2010). Costruire spazi di immaginazione auto/biografica. Quando i vissuti dei genitori diventano esperienza. Animazione sociale, 243, 34-41.

Formenti, L., \& West, L. (2018). Transforming perspectives in lifelong learning and adult education: A dialogue. Springer.

Gadamer, H. (1975). Hermeneutics and Social Science. Philosophy Social Criticism, 2(4), 307-316.

Green, L., Haddon, L., Livingstone, S., Holloway, D., Jaunzems, K., Stevenson, K., \& O’Neill, B. (2019). Parents' failure to plan for their children's digital futures (Media@LSE Working Paper Series, p. 21). LSE. http:// www.lse.ac.uk/media-andcommunications/assets/documents/research/working-paper-series/WP61.pdf

Henrich, J., Heine, S. J., \& Norenzayan, A. (2010). The weirdest people in the world? Behavioral and brain sciences, 33(2-3), 61-83. https://doi.org/10.1017/ S0140525X0999152X

Hine, C. (2014). Headlice eradication as everyday engagement with science: an analysis of online parenting discussions. Public Understanding of Science, 23(5), 574-591. https://doi.org/10.1177/0963662512453419

Holloway, D., \& Green, L. (2017). Mediated memory making: The virtual family photograph album. Communications, 42(3), 351-368. https://doi.org/10.1515/ commun-2017-0033

Holtz, P., Kronberger, N., \& Wagner, W. (2012). Analyzing Internet forums a practical guide. Journal of Media Psychology, 24(2), 55-66. https://doi. org/10.1027/1864-1105/a000062

Jarvis, P. (2012). Learning from everyday life. HSSRP, 1(1), 1-20.

Jewkes, Y., \& Wykes, M. (2012). Reconstructing the sexual abuse of children:'Cyber-paeds', panic and power. Sexualities, 15(8), 934-952. https://doi. org/10.1177\%2F1363460712459314

Jowett, A. (2015). A case for using online discussion forums in critical psychological research. Qualitative Research in Psychology, 12(3), 287-297. https://doi.org /10.1080/14780887.2015.1008906

Kruglanski, A. W., Dechesne, M., Orehek, E., \& Pierro, A. (2009). Three decades of lay epistemics: The why, how, and who of knowledge formation. European Review of Social Psychology, 20(1), 146-191. https:// doi.org/10.1080/10463280902860037

Landqvist, M. (2016). Sense and sensibility-online forums as epistemic arenas. Discourse, context \& media, 13, 98-105. https://doi.org/10.1016/j.dcm.2016.05.007

Leaver, T. (2015). Born digital? presence, privacy, and intimate surveillance. In J. Hartley \& W. QU (eds.), Re-Orientation: translingual transcultural transmedia. 
studies in narrative, language, identity, and knowledge (pp. 149-160). Fudan University Press.

Lee, E., Bristow, J., Faircloth, C., \& Macvarish, J. (2014). Parenting culture studies. Palgrave Macmillan.

Lindholm, L. (2017). "So now I'm panic attack free!" Response stories in a peer-topeer online advice forum on pregnancy and parenting. Linguistik Online, 87(8), 25-41. https://doi.org/10.13092/ lo.87.4171

Lupton, D., Pedersen, S., \& Thomas, G. M. (2016). Parenting and Digital Media: From the Early Web to Contemporary Digital Society. Sociology Compass, 10(8), 730-743. https://doi.org/10.1111/soc4.12398

Mascheroni, G., Ponte, C., \& Jorge, A. (eds.). Digital parenting. The challenges for families in the digital age (pp. 219-231). Nordicom, The Clearinghouse Yearbook.

Mascheroni, G. (2018). Datafied childhoods: contextualising datafication in everyday life. Current Sociology, 1-16. https://doi.org/10.1177/0011392118807534

Mezirow, J. (1991). Transformative dimensions of adult learning. Jossey-Bass.

Pedersen, S., \& Lupton, D. (2018). 'What are you feeling right now?' communities of maternal feeling on Mumsnet. Emotion, Space and Society, 26, 57-63. https://doi.org/10.1016/j.emospa.2016.05.001

Petti, L. (2011). Apprendimento informale in rete. Dalla progettazione al mantenimento delle comunità on line. Franco Angeli.

Riessman, C. K. (2008). Narrative methods for the human sciences. Sage.

Saldaña, J. (2009). The Coding Manual For Qualitative Researchers. Sage.

Siibak, A., \& Traks, K. (2019). The dark sides of sharenting. Catalan Journal of Communication \& Cultural Studies, 11(1), 115-121. https://doi.org/10.1386/cjcs.11.1.115_1

Shirani, F., Henwood, K., \& Coltart, C. (2012). Meeting the challenges of intensive parenting culture: gender, risk management and the moral parent. Sociology, 46(1), 25-40. https://doi. org/10.1177/0038038511416169

Smahel, D., Machackova, H., Mascheroni, G., Dedkova, L., Staksrud, E., Ólafsson, K., Livingstone, S. \& Hasebrink, U. (2020). EU Kids Online 2020: Survey results from 19 countries. EU Kids Online. https:// doi.org/10.21953/lse.47fdeqj01 ofo

Steinberg, S. B. (2017). Sharenting: children's privacy in the age of social media. Emory LJ, 66, 839.

Thomas, A., Stanford, P., \& Sarnecka, B. (2016). No child left alone: Moral judgments about parents affect estimates of risk to children. Collabra: Psychology, 2(1), 1-14. http://doi.org/10.1525/collabra.33
Tramma, S. (2019). L'educazione sociale. Editori Laterza.

Wenger, E. (2011). Communities of practice: A brief introduction. https://scholarsbank.uoregon.edu/ xmlui/bitstream/handle/1794/11736/A\%20brief\%20 introduction\%20to\%20CoP.pdf 